\title{
Double primary relaxation in a highly anisotropic orientational glass-former with low-dimensional disorder
}

\author{
Michela Romanini, ${ }^{\dagger}$ Maria Barrio, ${ }^{\dagger}$ Simone Capaccioli, ${ }^{\ddagger}$ Maria Dolores \\ Ruiz-Martin, ${ }^{\dagger}$ Roberto Macovez $^{\dagger}$ and Josep LI. Tamarit*,†
}

Grup de Caracterització de Materials, Departament de Física i Enginyeria Nuclear, ETSEIB, Diagonal 647, Universitat Politècnica de Catalunya, 08028 Barcelona, (Spain), and Dipartimento di Fisica, Università di Pisa, Largo B. Pontecorvo 3, I-56127 Pisa, Italy

E-mail: josep.lluis.tamarit@upc.edu

\begin{abstract}
The freezing of the cooperative reorientational motions in orientationally disordered (OD) molecular crystals marks the so-called "glassy" transition, which may be considered a lower-dimensional version of the structural glass transition. While structural glasses display both positional and orientational disorder, in fact, in orientational glasses the disorder involves exclusively the orientational degrees of freedom of the constituent molecules, while the molecular centres of mass form an ordered lattice. We report here on a glass-forming system with even less degrees of freedom, namely the OD phase of a dipolar benzene derivative, pentachloronitrobenzene $\left(\mathrm{C}_{6} \mathrm{Cl}_{5} \mathrm{NO}_{2}\right)$. We probe the orientational dynamics of PCNB as a function of temperature and pressure

\footnotetext{
*To whom correspondence should be addressed

${ }^{\dagger}$ Universitat Politècnica de Catalunya

$\ddagger$ Università di Pisa
} 
by means of dielectric spectroscopy at normal and high pressure and high-pressure density measurements, and show that the system exhibits a double primary relaxation feature associated with two distinct motions of the molecular dipole moment. After ruling out an interpretation in terms of primitive or intramolecular relaxations, we discuss an assignment of the double relaxation feature based on the material's anisotropy and on the comparison with discotic liquid crystals.

\section{Introduction}

The glass transition in structural glass formers is associated with the freezing of rototranslational (diffusional) degrees of freedom. The glass state is obtained when, upon cooling or pressurizing fast enough to avoid the transition to the crystalline state, a liquid gradually freezes, into an amorphous phase in which the molecular motions are quenched while the structural disorder of the liquid is retained. ${ }^{1-4}$ A glass-like dynamics may appear also when a translational long-range order exists. In fact, many molecular systems form phases which are characterized by translational order and at the same time by orientational disorder. In these orientationally disordered (OD) crystals the average position of the centres of mass of the molecules form a lattice while the molecular orientations are dynamically disordered. ${ }^{5-7}$ The reorientational motions may freeze upon cooling or pressurizing, resulting in an orientational glass: OD phases therefore exhibits a phenomenology that is analogous to that of structural glass formers. ${ }^{4,8-22}$ Due to the large number of degrees of freedom of its constituent molecules, molecular condensed-matter systems are characterized by a rich variety of dynamic processes and phases. In molecular materials forming structural or orientational glasses, the most important dynamics is the cooperative motion of the molecules, referred to as primary relaxation or $\alpha$ process, whose freezing marks the transition to the glass state. The main difference between orientational and structural glasses is that in the former the freezing involves exclusively the rotational degrees of freedom of the molecules, while in the

latter all six molecular degrees of freedom (i.e., both orientational and translational ones) 
are frozen. Orientational glasses are therefore systems with fewer degrees of freedom than structural glasses. This simplification, together with the fact that many OD phases are characterized by a high-symmetry crystal lattice, makes orientationally disordered phases a model playground to investigate the nature of the glass transition, which is one of the unsolved problems in condensed-matter physics. We discuss in this contribution a glassforming system with yet lower number of degrees of freedom than conventional OD crystals, namely the layered solid phase of the hexasubstituted pentachloronitrobenzene derivative $\left(\mathrm{C}_{6} \mathrm{Cl}_{5} \mathrm{NO}_{2}\right.$, hereinafter PCNB). The low-dimensionality arises because these quasi-planar benzene derivatives form a stacked structure of parallel planes in which the molecules can only rotate around a fixed (six-fold-like) axis orthogonal to the planes. ${ }^{23,24}$

Molecular dynamic processes in glass formers other than the $\alpha$ relaxation are termed "secondary relaxations", and are usually characterized by shorter relaxation time than the primary process associated with the glass transition. Secondary relaxations in OD phases may have different origins; for example, the constituent molecules generally exhibit intramolecular dynamics, such as conformational motions or vibrations of inter-atomic bonds; in other cases a single-molecule precursor of the $\alpha$ process is observed, which is called Johari-Goldstein relaxation and is usually interpreted in the framework of the coupling model. ${ }^{25-27}$ The spectral landscape of secondary relaxations of structural glass formers is further enriched by the existence of the so-called excess wing (EW), that is, of an excess dielectric loss on the highfrequency side of the $\alpha$-relaxation process. ${ }^{17,18,28,29}$ Based on comparative studies of several structural glass formers, some authors ${ }^{30}$ have proposed that the EW could actually be a nonresolved secondary relaxation process hidden below the high-frequency wing of the primary $\alpha$-relaxation. In recent work on structural glasses, application of high pressure confirmed that the EW is indeed a "submerged" secondary relaxation. ${ }^{31}$ The frequency separation between the $\alpha$ relaxation process and such secondary relaxation has been shown to be invariant under isochronal conditions within the temperature-pressure diagram. ${ }^{32}$ These studies have thus concluded that the EW is a "precursor" Johari Goldstein relaxation, as shown e.g. for 
the case of glycerol, ${ }^{31}$ ethanol, ${ }^{17}$ and propylene glycol oligomers. ${ }^{33}$

As far as orientationally disordered materials are concerned, an earlier work ${ }^{18}$ based on the comparison of several OD phases concluded that the EW is absent and thus not a universal feature of such glass formers. However, few years later the same authors reported ${ }^{19}$ the possible existence of an EW in PCNB. The phase behaviour of this molecule, which forms a layered structure of triangular planes, has been extensively studied. ${ }^{19,23,24,34-41}$ A glass transition was observed by adiabatic calorimetry at approximately $185 \mathrm{~K}$, which was assigned to the freezing of the orientational disorder within the hexagonal planes. ${ }^{37}$ Similar results were found by Correia et al. by DSC and thermally stimulated currents, with a reported transition temperature of $191 \mathrm{~K} .{ }^{38,39}$ Here, we show by means of broadband dielectric spectroscopy (BDS) that such phase is characterized by a double primary (collective) relaxation which is related to the low-dimensional character of the system and which results in a spectral landscape reminiscent of $\alpha$ relaxations characterized by an excess wing. In order to investigate the connection between the dielectric and structural properties, we have characterized the material both by X-ray diffraction and BDS, as a function both of temperature and pressure. We find that the excess wing is not a Johari-Goldstein precursor, but is in fact a separate relaxation than the $\alpha$ process, and propose a microscopic interpretation for the origin of both relaxation processes based on the material's anisotropy.

\section{Results and discussion}

PCNB displays, in the condensed phase, a stacked structure of molecular planes in which the PCNB molecules lie flat on each plane occupying the sites of a triangular lattice. ${ }^{23,24} \mathrm{~A}$ Monte Carlo computer simulation modelling available single-crystal diffuse X-ray scattering data $^{23}$ has shown that the solid phase of PCNB, which melts at ca. $417 \mathrm{~K}$, is rhombohedral (space group $R \overline{3}$ ) with each site of the crystal containing a molecule in one of the six possible different orientations in the plane, each occupied with equal probability. Singe-crystal 
neutron diffraction data has confirmed the $1 / 6: 5 / 6 \mathrm{NO}_{2}: \mathrm{Cl}$ occupation factors and shown that the $\mathrm{O}$ atoms of the $\mathrm{NO}_{2}$ group are rotated out of the benzene plane in such a way that both planes are $74^{\circ}$ apart. ${ }^{24}$ The latter study has moreover revealed that the $U_{33}$ components (along the $c$ axis) of the tensor for the anisotropy displacement parameters are much larger than the $U_{11}$ and $U_{22}$ principal coefficients (of the (001) in-plane displacements) whatever the temperature and for all atoms, but specially for $C l, N$ and $O$. Motivated by these findings, we measured the temperature dependence of the lattice parameters by means of X-ray powder diffraction. The diffraction pattern at $300 \mathrm{~K}$ matches the pattern simulated using the structural data from Thomas et al. ${ }^{23}$ The similarity between the diffraction profiles acquired at distinct temperatures (see supporting information) confirms that no structural change takes place in the 150-300 K range. We used this data as starting point for the refinement of the lattice parameters by means of the Pattern Matching option of the Full Prof program. ${ }^{42}$ Figure 1 shows the temperature-dependence of the lattice parameters, and of the unit cell volume, from below the glass transition to room temperature. The continuous variation of the lattice parameters (and, as a consequence, of the volume per molecule) together with the discontinuity in the volume expansivity (defined as $\alpha_{V}=1 / V(\partial V / \partial T)_{P}$ ) are irrefutable signatures of a glass transition, which takes place at approximately $187 \mathrm{~K}$.

From Figure 1 one gathers that, as the temperature decreases, the lattice contracts more in the $c$ direction $\left(\alpha_{c}\left(T \geq T_{g}\right)=1 / c(\partial c / \partial T)_{P}=1.45 \cdot 10^{-3} K^{-1}\right)$ than within the (001) planes $\left(\alpha_{a}\left(T \geq T_{g}\right)=1 / a(\partial a / \partial T)_{P}=4.0 \cdot 10^{-5} K^{-1}\right)$. Also, the kink at the glass transition in the temperature dependence of the lattice parameter $c$ is much more pronounced than that of the parameter $a$, the change of the slope being more than one order of magnitude different. In other words, the inter-plane structural parameter is much more affected by changes of temperature and by the kinetic transition than any intraplane distance. This is obviously directly related with the larger values of the $U_{33}$ component compared to $U_{11}$ and $U_{22} \cdot{ }^{24}$

Figure 2 displays several representative spectra of the imaginary part of the complex permittivity of PCNB, measured both isobarically (a), isothermally (b), and a comparison 


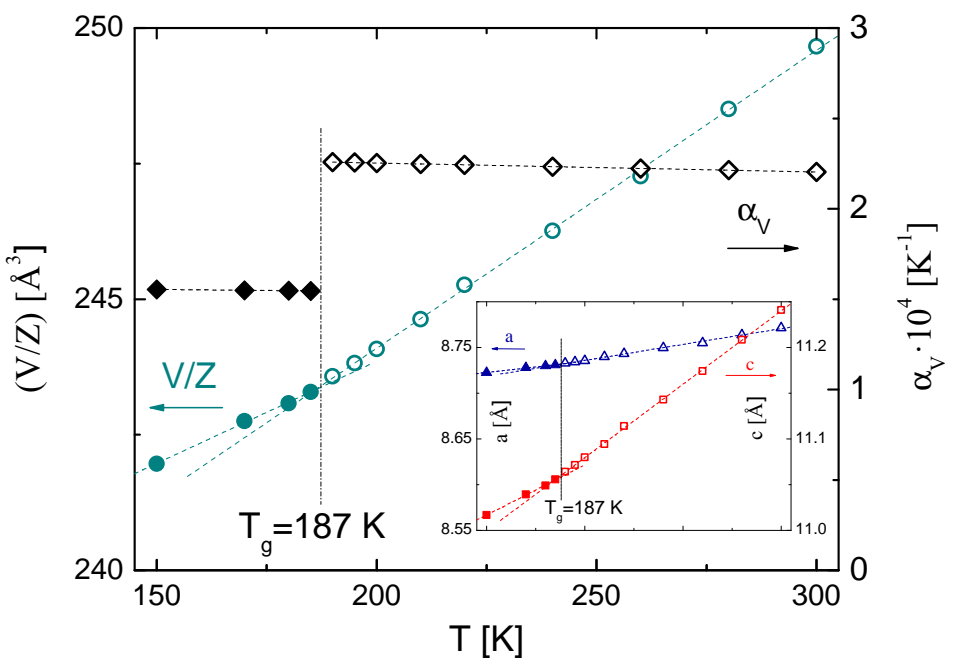

Figure 1: Molecular cell volume normalized to the number of molecules $Z$ in the unit cell (open and closed circles) and thermal expansivity (open and closed diamonds) of PCNB as a function of temperature. Inset: lattice parameters $a$ (open and closed triangles) and $c$ (open and closed squares).

at fixed relaxation time of the $\alpha$ process (that is, isochronically for various (P,T) pairs, (c)). Details of the experimental device can be found in ref. ${ }^{5}$ and in the Supp. Info. In all spectra a clear relaxation peak is observed, displaying continuous temperature and pressure shifts characteristic of the freezing of the molecular dynamics on cooling and pressurizing. The frequency position and temperature dependence of the main peak are in agreement with early normal pressure dielectric studies at on PCNB. ${ }^{34-36}$ In addition to a well-pronounced main peak (hereafter $\alpha$ relaxation), an excess wing (hereafter called $\alpha^{\prime}$ process) is clearly visible as a shoulder to the main peak. The fact that this loss process appears as a shoulder indicates that its dielectric strength is lower than that of the main loss (see also below). Panels (a) and (b) of Figure 2 highlight the need of two dipolar processes (displayed as dotted lines) for modelling the dielectric spectra. It should be noticed that in previous work ${ }^{19,40}$ only one HN function was employed to fit the loss curves, and this resulted in a slight discrepancy between the experimental data and the fit function in the high-frequency range of the spectra.

Figure 2(c) shows that the shape of the loss spectra is almost the same for different combinations of $\mathrm{T}$ and $\mathrm{P}$ chosen so as to maintain the same value of the relaxation time for 
the $\alpha$ process, namely at the isochronal value $\log \left(f_{\text {max }_{\alpha}}\right)=2.4$ corresponding to ca. $0.6 \mathrm{~ms}$. The figure evidences that the relative position and strength of both processes and the overall shape of the spectra are unchanged despite the large range of temperatures and pressures spanned by the experiments. The rough superposition of all isochronal spectra into a single master curve indicates that the two dynamic processes have similar dependence upon the thermodynamic parameters, highlighting a strong correlation between both relaxations. This rationalizes the fact that the $\alpha^{\prime}$ process was initially considered to be an excess wing. ${ }^{19}$

It is worth noting that a relaxation phenomenology similar to PCNB has been reported in related molecular systems, namely discotic liquid crystals consisting of phenyl-based, rigid disk-shaped aromatic flat cores functionalized with flexible polar sidegroups. ${ }^{43-45}$ These materials, whose constituent molecules can be considered to be larger "cousins" of the hexasubstituted benzenes, display in fact two dielectric relaxations with different characteristic times but virtually identical temperature dependencies. ${ }^{44}$ Also in the case of discotic liquid crystals, the lower-frequency relaxation corresponds to the main $\alpha$ process, as discernible from the position of the loss maximum at the corresponding $T_{g}$ (determined from calorimetry measurements), and the second $\left(\alpha^{\prime}\right)$ process at higher frequency displays lower dielectric strength than the first one.

All measured PCNB spectra were fitted using a combination of a Havriliak-Negami (HN) function for the main relaxation $(\alpha)$ together with a Cole-Cole $(\mathrm{CC})$ function for the shoulder relaxation $\left(\alpha^{\prime}\right) .{ }^{46}$ The resulting fits are shown as continuous lines in Figure 2 ; it can be seen in all three panels that the model provides quite accurate fits. A reasonable physical consistency was found for the fit parameters. The shape parameters $\alpha_{H N}$ and $\beta_{H N}$ of the HN function and the exponent $\alpha_{C C}$ of the CC function all lay in the range between 0.61 and 1 .

The characteristic relaxation times of both processes, as obtained from the fitting procedure, are plotted in Figure 3 both as a function of temperature at normal pressure (upper panel) and as a function of pressure at constant temperature (lower panel). It can be observed that the two relaxations $\left(\alpha\right.$ and $\left.\alpha^{\prime}\right)$ have similar but not identical dependencies on 

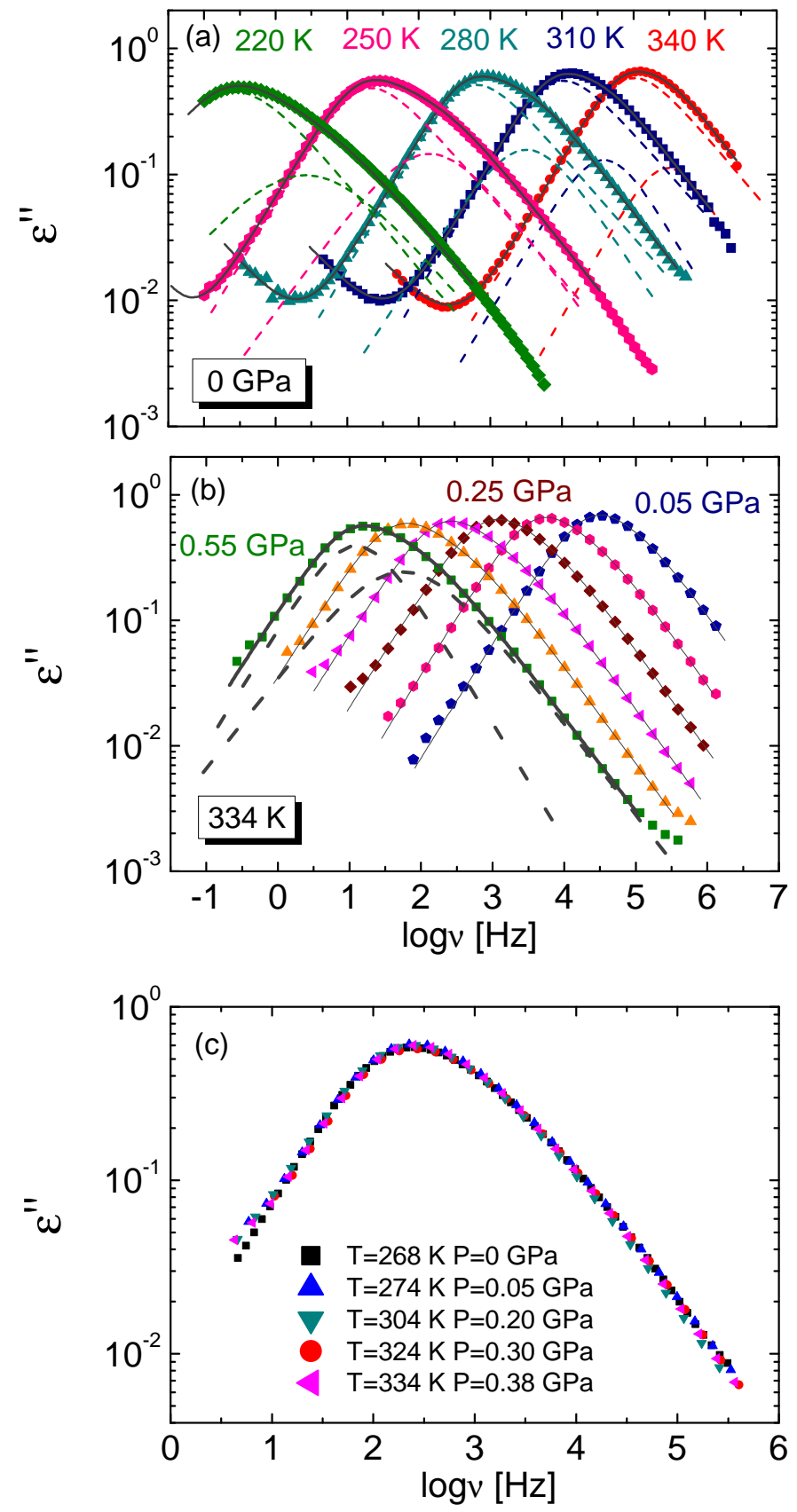

Figure 2: Dielectric loss curves acquired at ambient pressure at the indicated temperatures (a) and at constant temperature $(334 \mathrm{~K})$, acquired between 0.05 and 0.55 GPa every 0.05 GPa (b). Coloured markers are experimental points, while the solid lines represent the fits of the spectra as the sum of two components, shown explicitly with dotted lines for all temperatures in (a), and at $0.55 \mathrm{GPa}$ in (b). (c) Superposition of loss spectra measured for a combination of temperatures and pressures displaying the main relaxation peak at the same frequency $f_{\max }=251 \mathrm{~Hz}\left(\tau_{\max } \approx 0.6 \mathrm{~ms}\right)$. 

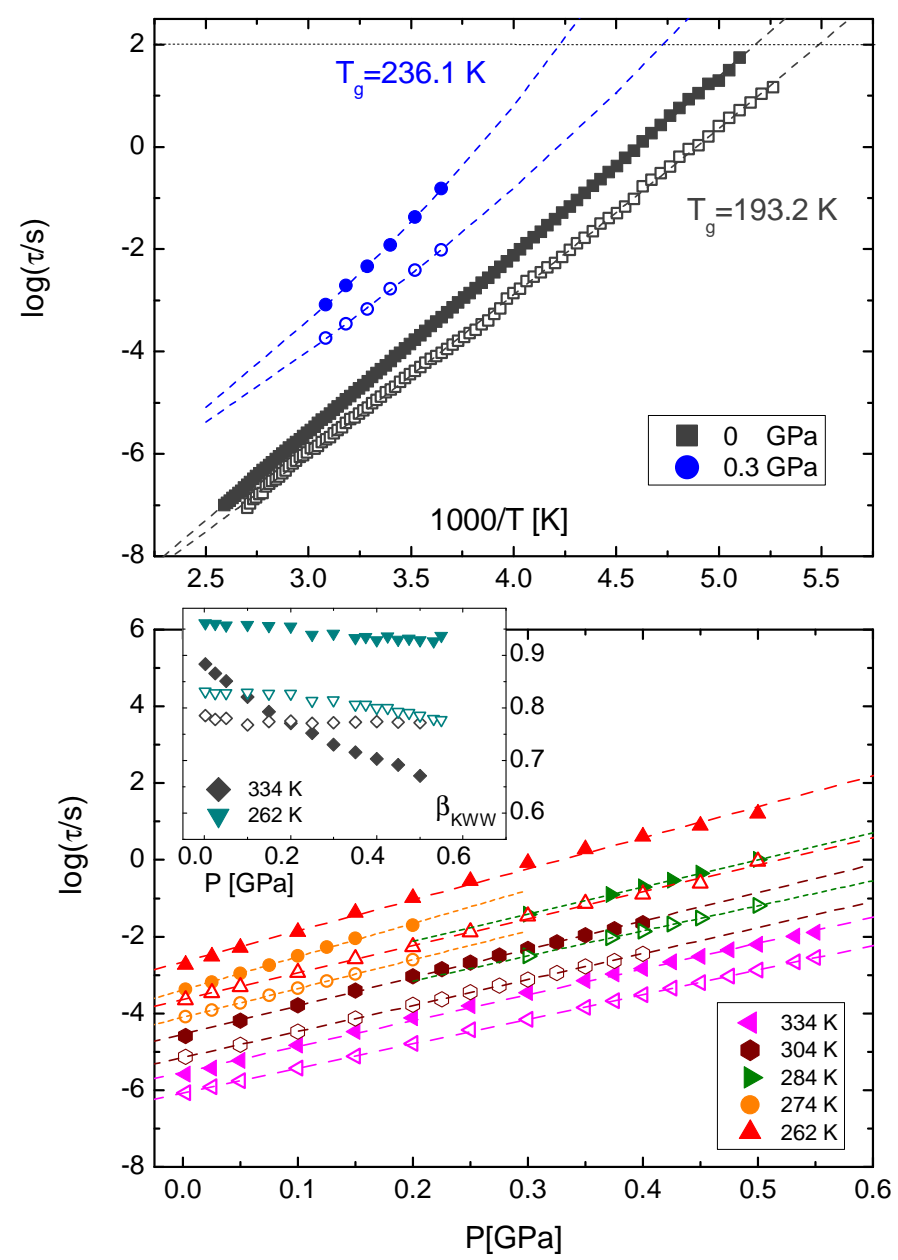

Figure 3: Relaxation times for the $\alpha$ (closed symbols) and $\alpha^{\prime}$ (open symbols) relaxations as a function of the reciprocal temperature for isobaric measurements (upper panel), and as a function of pressure for isothermal measurements (lower panel). The constant temperatures or pressures employed are indicated in the legends. Inset in the lower panel: values of the $\beta_{K W W}$ exponent for the $\alpha$ (closed symbols) and $\alpha^{\prime}$ (open symbols) processes at fixed temperature as a function of pressure. 
Table 1: Values of the glass transition temperature $\left(T_{g}\right)$ and pressure $\left(P_{g}\right)$, fragility $(m)$ and activation volume $\left(\Delta V^{\#}\right)$ for the measurements at constant pressure and temperature shown in Figure 2.

\begin{tabular}{|cccc|}
\hline $\begin{array}{c}\mathrm{P} \\
(\mathrm{GPa})\end{array}$ & process & $\begin{array}{c}T_{g} \\
(\mathrm{~K})\end{array}$ & $\mathrm{m}$ \\
\hline 0 & $\alpha$ & $193.2 \pm 0.1$ & $18.2 \pm 0.1$ \\
& $\alpha^{\prime}$ & & $18.6 \pm 0.1$ \\
0.3 & $\alpha$ & $236.1 \pm 0.2$ & $22.5 \pm 0.1$ \\
& $\alpha^{\prime}$ & & $19.6 \pm 0.1$ \\
\hline $\mathrm{T}$ & process & $P_{g}$ & $\Delta V^{\#}$ \\
$(\mathrm{~K})$ & & $(\mathrm{GPa})$ & $\left(\mathrm{cm}^{3} / \mathrm{mol}\right)$ \\
\hline 334 & $\alpha$ & $1.12 \pm 0.01$ & $43.0 \pm 0.4$ \\
& $\alpha^{\prime}$ & & $40.9 \pm 0.1$ \\
304 & $\alpha$ & $0.89 \pm 0.01$ & $42.9 \pm 0.5$ \\
& $\alpha^{\prime}$ & & $39.4 \pm 0.1$ \\
284 & $\alpha$ & $0.78 \pm 0.01$ & $38.6 \pm 0.2$ \\
& $\alpha^{\prime}$ & & $35.5 \pm 0.3$ \\
274 & $\alpha$ & $0.62 \pm 0.02$ & $45.4 \pm 0.9$ \\
& $\alpha^{\prime}$ & & $39.3 \pm 0.1$ \\
262 & $\alpha$ & $0.58 \pm 0.02$ & $40.6 \pm 0.9$ \\
& $\alpha^{\prime}$ & & $35.2 \pm 0.5$ \\
\hline
\end{tabular}

$\mathrm{T}$ and $\mathrm{P}$. This shows that the $\alpha^{\prime}$ process is not an excess wing, but a separate dynamical process than the $\alpha$ relaxation. The values of the glass-transition temperatures and pressures, fragility and activation volume are reported in Table 1 for all measurements. The low fragility values obtained from isobaric measurements indicate that PCNB is a quite a strong glass former.

From the comparison of temperature- and pressure-dependent measurements it is possible to determine whether the molecular dynamics is dominated (limited) by the thermal energy or by the available free volume. In particular, one can use the ratio between the activation enthalpy at constant volume $\left(E_{V}=R\left[\partial \log \tau / \partial T^{-1}\right]_{V}\right)$ and the activation enthalpy at constant pressure $\left(E_{P}=R\left[\partial \log \tau / \partial T^{-1}\right]_{P}\right)$ : if this ratio is between 0 and 0.5 , then the dynamics is volume-dominated; if it this between 0.5 and 1 the dynamics is temperature-dominated. ${ }^{47}$ We have calculated both activation enthalpies by considering the isothermal data presented in the lower panel of Figure 3. The enthalpy at constant pressure was obtained plotting 
the relaxation times of both $\alpha$ and $\alpha^{\prime}$ relaxations obtained in the isothermal measurements at pressures between normal pressure and $0.6 \mathrm{GPa}$ every $0.1 \mathrm{GPa}$ as a function of the corresponding temperatures, and then calculating the activation enthalpy as the slope of the linear fit of such plot. For the enthalpy at constant volume, we plotted the relaxation times as a function of the specific volume at given $(\mathrm{P}, \mathrm{T})$ values. The specific volume was calculated by means of the Tait equation ${ }^{48}$ resulting from the fit (see Supp. Info.) of pressure-volume-temperature measurements on PCNB, carried out with the experimental setup described in ref. ${ }^{49}$ The outcome is shown in the inset to Figure 4 . The enthalpy at constant volume was then obtained by plotting the relaxation times of both $\alpha$ and $\alpha^{\prime}$ relaxations at three fixed specific volumes $\left(\mathrm{V}=0.485,0.500\right.$ and $\left.0.505 \mathrm{~cm}^{3} / \mathrm{mol}\right)$ as a function of the corresponding temperatures, and then calculating the slope of the linear fit of such plot. The ratio $E_{V} / E_{P}$ is found to lie between 0.51 and 0.77 for both the $\alpha$ and $\alpha^{\prime}$ processes, which would indicate that both dynamics are temperature-dominated. Moreover, the ratio decreases with increasing pressure, as expected because a pressure increase entails a increase of the density and therefore a higher activation volume barrier.

It is possible to obtain a "thermodynamic" scaling of all the relaxation time data $\tau_{\alpha}(T, P)$ as a function of a single scaling variable $1000 / T V^{\gamma}$, where $\gamma$ is a material constant. ${ }^{50}$ The result of the thermodynamic scaling of the relaxation time data is shown in Figure 4 . We found that the $\alpha$ and $\alpha^{\prime}$ relaxations scale with slightly different factors, namely $\gamma_{\alpha}=7.60 \pm$ 0.02 and $\gamma_{\alpha^{\prime}}=7.81 \pm 0.01$ respectively. This again confirms that the $\alpha$ and $\alpha^{\prime}$ processes corresponds to different dynamics.

Given the rigidity of the hexagonal carbon backbone of the PCNB molecule and of the bonds to the side groups $\mathrm{Cl}$ and $\mathrm{NO}_{2}$, the $\alpha^{\prime}$ process cannot be attributed to the motion of an intramolecular dipole moment behaving independently from the main dipole moment of the molecule. The motion of $C l$ atoms with respect to the carbons can only give rise to vibrational modes in the IR region of the electromagnetic spectrum. If intramolecular motions of the $\mathrm{NO}_{2}$ sidegroups took place, given the small mass of the $\mathrm{NO}_{2}$ group they would 


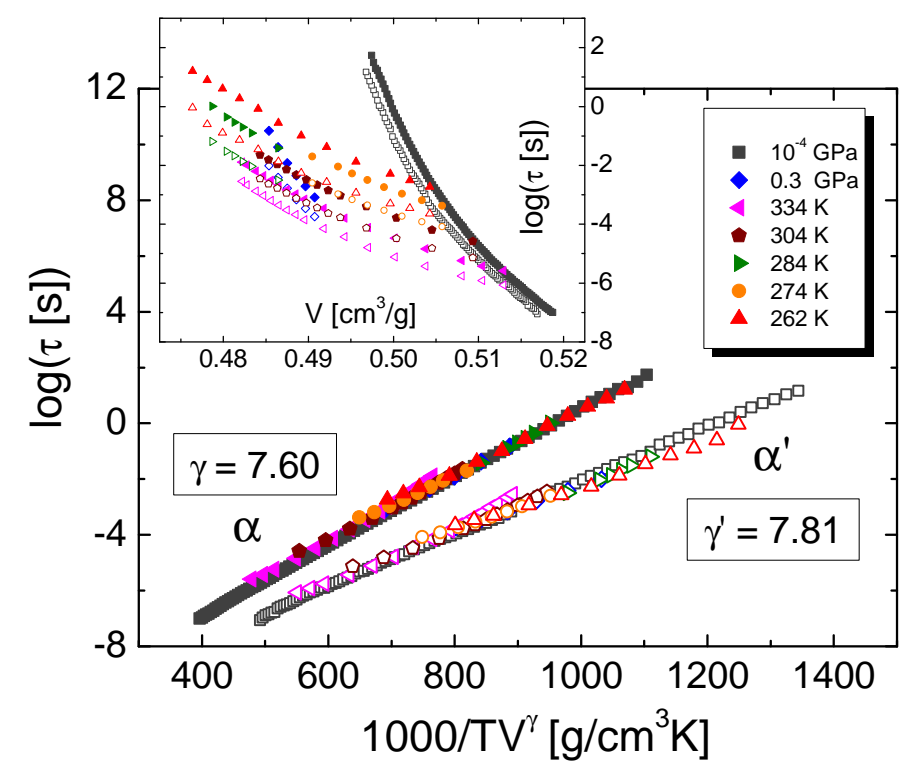

Figure 4: Thermodynamic scaling of the relaxation times for the $\alpha$ (closed symbols) and $\alpha^{\prime}$ (open symbols) processes as a function of the scaling quantity 1000/TV ${ }^{\gamma}$. Inset: relaxation times of both $\alpha$ (closed symbols) and $\alpha^{\prime}$ (open symbols) processes as a function of the specific volume, as calculated at the appropriate $(\mathrm{P}, \mathrm{T})$ values by means of the Tait equation (see supporting information).

also be much faster than either the $\alpha$ and $\alpha^{\prime}$ processes, at least like the $\beta$ relaxation reported in an earlier study at much lower temperature (between 140 and $170 \mathrm{~K}$, i.e., clearly below $\left.T_{g}\right)$ and with relaxation times far away from those here reported $\left(\tau_{\beta} \approx 1 \mathrm{~ms}\right.$ at $\left.140 \mathrm{~K}\right) .{ }^{19}$ As mentioned previously, the $\mathrm{NO}_{2}$ group is occupationally disordered and displays large displacements and torsional vibrations accompanying whole-molecule librations; however, according to the diffraction studies ${ }^{23}$ it doesn't display rotations around the C-N axis. Hence the $\alpha^{\prime}$ process cannot correspond to a reorientation of the $\mathrm{NO}_{2}$ dipole independent of that of the rest of the molecule. ${ }^{24}$

At the same time, the $\alpha^{\prime}$ process cannot be identified as the primitive (Johari-Goldstein) precursor relaxation of the $\alpha$ process. In fact, a correlation between the $\alpha$ - and $\beta_{J G}$-relaxation times has been experimentally proven, based on empirical findings on a large number of glass formers. ${ }^{27}$ According to these studies, the primitive JG relaxation time $\left(\tau_{J G}\right)$ is related to the $\alpha$-relaxation time $\left(\tau_{\alpha}\right)$ as: 


$$
\tau_{J G}=\left(t_{c}\right)^{1-\beta_{K W W}} \tau_{\alpha}^{\beta_{K W W}}
$$

Here, $t_{c}$ is the crossover time which marks the onset of the many-body dynamics (typically $t_{c} \approx 2$ ps in molecular glass-formers) and $\beta_{K W W}$ is the stretched exponent of the KohlrauschWilliams-Watts function representing the $\alpha$-relaxation process. By using, for example, the value of $\tau_{\alpha}$ of Figure $2(\mathrm{c})\left(\tau_{\alpha}=0.63 \cdot 10^{-3} \mathrm{~s}\right)$ and the corresponding $\beta_{K W W}$ value $(\approx 0.90)$, the calculated $\tau_{J G}$ is found to be two decades smaller than $\tau_{\alpha^{\prime}}$.

It follows that the origin of the $\alpha^{\prime}$ loss cannot be a secondary relaxation of any kind, but must instead stem from the motion of the PCNB molecule as the primary $\alpha$ relaxation (as also testified by their similar behaviour, see Figure 2. To identify the origin of the $\alpha^{\prime}$ feature, it is helpful to compare our BDS results with structural data and with dielectric relaxation results on discotic liquid crystal systems. The dipole moment of the PCNB molecule $\left(\mu=2.33 \mathrm{D}^{34}\right)$ is determined to a large extent by that of the $\mathrm{NO}_{2}$ sidegroup. In the solid phase, the $\mathrm{NO}_{2}$ group of a PCNB molecule, which, as mentioned, is tilted with respect to the (001) plane, points towards a $C-C l$ bond of a molecule in the next layer and away from its $\mathrm{NO}_{2}$ group (both molecules being related by an inversion centre), whereas the other chlorines of both molecules are bent towards each other. As shown by Monte Carlo simulations, ${ }^{23}$ these correlations are accompanied by large atomic displacements compared with the spacing between intermolecular layers along the $c$ axis $(3.75 \AA)$, which imply a high level of molecular distortion. The local displacements evidenced by the analysis of the diffraction data, ${ }^{23}$ result in an increase of the intermolecular $\mathrm{NO}_{2} \cdots \mathrm{NO}_{2}$ and $\mathrm{NO}_{2} \cdots \mathrm{Cl}$ nearest-neighbour distances and a decrease of the $C l \cdots C l$ ones.

These findings suggest that intermolecular interactions mediated by $\mathrm{NO}_{2}$ and $\mathrm{Cl}$ groups are responsible for strongly correlated displacements and distortions which prevent the formation of a fully ordered crystalline structure. Hence the at first sight flat, 2D-like benzene derivative is in fact a 3D-like molecule with strong fluctuations. Because of the intermolecular interactions involving the $\mathrm{NO}_{2}$ groups, the molecular dipole cannot lie exclusively on the 
(001) layers but must possess a component along the $c$ axis. The molecular dipole of PCNB (largely due to the nitro group) has therefore a larger component orthogonal to $c$, and a shorter one along $c$. The reported molecular displacements imply that both components, and in particular the one parallel to $c$, exhibit very large fluctuations; due to steric effects, these fluctuations are strongly correlated between one molecule and the next, i.e. they are necessarily highly cooperative.

The above discussion implies that the in-plane and out-of-plane anisotropy of PCNB is reflected both in the microscopic dipole moments and in their mutual interactions: the molecular dipoles have two non-zero (in-plane and out-of-plane) components, and they also display very anisotropic coupling in the in-plane and out-of-plane directions. Such a situation is reminiscent of that of columnar hexagonal phases of discotic liquid crystals of disk-shaped molecules with disordered flexible sidegroups (alkyl or other substituent chains). These molecules posses dipole moments (associated with the flexible sidegroups) which are not oriented parallel to the molecular disk, and which display highly anisotropic interactions in two directions, namely within the same column and between adjacent column. ${ }^{43-45}$ Discotic liquid crystals also display a "double" primary relaxation with similar temperature dependence as found in PCNB. In other words, the two systems, PCNB and discotic liquid crystal, share both the same degree of anisotropy and a similar phenomenology.

In light of this, we are lead to assign the $\alpha$ and $\alpha^{\prime}$ relaxations to distinct molecular dynamic processes involving the in-plane and out-of-plane components of the dipole moment of the PCNB molecule. In particular, we ascribe the main $\alpha$ process to the reorientational motions of the molecular dipole within the (001) planes, i.e. to molecular reorientational jumps which modify directly the in-plane component of the dipole moment. As mentioned above the larger dipole component lies on the (001) plane and consequently its reorientational dynamics must exhibit higher dielectric strength. The second process, $\alpha^{\prime}$, slightly faster than the $\alpha$ process, is instead connected with the relaxation of the dipole component fluctuating around the $c$ axis, i.e., with "shaking" motions of the PCNB molecules that leave basically 
invariant the in-plane component, but which affect strongly the out-of-plane contribution of the molecular dipole (significantly shorter than the in-plane one). The relative dielectric strength of the $\alpha$ and $\alpha^{\prime}$ processes corroborates this assignment, since the strength of the main $\alpha$ process is larger than that of the $\alpha^{\prime}$ process for all values of the thermodynamic parameters. We moreover observed (see supporting information) that the strength of the $\alpha^{\prime}$ process diminishes with respect to that of the $\alpha$ process with decreasing temperature. This effect is much more accentuated at high pressure and low temperatures. Since the increase of pressure or the reduction of temperature have a much stronger effect on the $c$ lattice parameters (see the anisotropic thermal expansion of lattice parameters in Figure 1), the assignment of the $\alpha^{\prime}$ relaxation to the dynamics along the $c$ direction rationalizes the pronounced effect of $P$ (and $T$ ) variations on this relaxation feature.

Also the fact that the $\alpha^{\prime}$ process is observed at higher frequency (that is, at shorter times) can be rationalized with our assignment. In fact, the dipole component in the plane relaxes by whole-molecule rotations inside the plane; these are limited by the steric hindrance of the sidegroups of neighbouring PCNB molecules in the same plane (which is large as evidenced by the small expansivity in the plane). In contrast, the vertical component of the dipole can relax only through the fluctuations of the out-of-plane dipole component and is therefore affected by the smaller inter-plane hindrance associated with the soft interactions along $c$ (as evidenced by the large expansivity along $c$ ). ${ }^{51}$ The fact that both $\alpha$ and $\alpha^{\prime}$ processes are due to the motion of the same dipole moments justifies our choice of the symbol $\alpha^{\prime}$ to label the faster relaxation and the phrasing "double primary relaxation" to refer to the dielectric response of $\mathrm{PCNB}$.

As stated in the introduction, PCNB can be considered a low-dimensional analogue of a plastic crystal. While it may be expected to display a less complex dielectric spectrum than plastic crystalline phases due to the fewer degrees of freedom, the low-dimensional PCNB glass-former actually exhibits a richer landscape, with two distinct whole-molecule (primary) relaxations. The origin of the more complex dielectric response is ultimately the 
fact that the low-dimensionality of the system really entails a pronounced anisotropy, leading to a distinct behaviour of the two degrees of freedom (molecular dipole components) of the PCNB molecules.

We finally discuss the shape of each spectral component. The pressure dependence of the stretched $\beta_{K W W}$ exponents of both relaxations are displayed in the inset to Figure 3 at two fixed temperatures. For the $\alpha$ process, the $\beta_{K W W}$ parameter (obtained from the parameters of the $\mathrm{HN}$ function as $\left.\left(\alpha_{H N} \beta_{H N}\right)^{1 / 1.23}\right)$ decreases as the pressure increases, this behaviour being more relevant at high-temperature and, as usual, it increases with temperature at constant pressure. The $\beta_{K W W}$ for the $\alpha^{\prime}$ process (obtained from the exponent of the ColeCole function as $\left.\left(\alpha_{C C}\right)^{1 / 1.23}\right)$ decreases slowly with the decrease of temperature and is almost invariant with pressure. It should be noticed that the $\beta_{K W W}$ values for the $\alpha^{\prime}$ process are noticeably smaller than those for the $\alpha$ process at high temperature as well as at low pressure, which entail that the $\alpha^{\prime}$ relaxation is more cooperative than the $\alpha$ relaxation except at very high pressure and low temperature. The pronounced cooperativity of the $\alpha^{\prime}$ relaxation again confirm its "primary" nature.

To conclude, this study is, to the best of our knowledge, the first one on the slow dynamics of statistical disordered structures under simultaneous variations of temperature and pressure through dielectric spectroscopy. Despite the long-range translation order of PCNB, its rotational dynamic properties display very strong similarities to those of structural glassformers: (i) the dependence of the glass transition on pressure is similar to that of van der Waals molecular liquids; (ii) the overall rotational relaxation function is invariant if considered at isochronal conditions. The low-dimensionality and anisotropy of the system leads to a more complex scenario than that of the (more isotropic) plastic crystalline phases, leading to a double primary relaxation feature which is reminiscent of the excess wing of some glass-forming supercooled liquids.

For the studied temperature and pressure ranges, the dynamics reveals two relaxation processes very close in time scale which are attributed, respectively, to the in-plane reori- 
entational motions of the molecules within the (001) plane ( $\alpha$ process), which are frozen at $T_{g}=193 \mathrm{~K}$, and the out-of-plane faster contribution, associated with the relaxation of the residual dipole fluctuating around the $c$ hexagonal axis ( $\alpha^{\prime}$ process). The strength of the $\alpha^{\prime}$ process is lower since the component parallel to the $c$ axis is smaller than that orthogonal to it, and the dynamics of both processes is different due to the anisotropy of intermolecular interactions.

\section{Acknowledgement}

This work was supported by the Spanish Ministry of Science and Innovation (Grant FIS201124439) and the Catalan Government (Grant 2014SGR-581). One of us (SC) thanks the

Spanish Ministry (SB2009-0139) for an invited position at the Universitat Politécnica de Catalunya.

\section{Supporting Information Available}

Description of the experimental details, example of a X-ray powder diffraction spectrum and fit based...., experimental pressure-volume-temperature data and fit by means of the Tait equation, plot of the ratio between the dielectric strength of the $\alpha$ - and $\alpha^{\prime}$-relaxation process as a function of pressure.

This material is available free of charge via the Internet at http://pubs.acs.org/.

\section{References}

(1) Berthier, L.; Biroli, G. Rev. Mod. Phys. 2011, 83, 587.

(2) Debenedetti, P. G.; Stillinger, F. H. Nature 2001, 410, 259.

(3) Angell, C. A.; Ngai, K. L.; McKenna, G. B.; McMillan, P. F.; Martin, S. W. J. Appl. Phys. 2000, 88, 3113. 
(4) Lunkenheimer, P.; Schneider, U.; Brand, R.; Loidl, A. Contemp. Phys. 2000, 41, 15.

(5) Romanini, M.; Negrier, P.; Tamarit, J. L.; Capaccioli, S.; Barrio, M.; Pardo, L. C.; Mondieig, D. Phys. Rev. B 2012, 85, 134201.

(6) Macovez, R.; Goldoni, A.; Petaccia, L.; Marenne, I.; Brühwiler, P. A.; Rudolf, P. Phys. Rev. Lett. 2008, 101, 236403.

(7) Haida, O.; Suga, H.; Seki, S. Bull. Chem. Soc. Jpn. 1977, 50, 802.

(8) Jiménez-Ruiz, M.; Criado, A.; Bermejo, F. J.; Cuello, G. J.; Trouw, F. R.; FernándezPerea, R.; Löwen, H.; Cabrillo, C.; ; Fischer, H. E. Phys. Rev. Lett. 1999, 83, 2757.

(9) Criado, A.; Jimenez-Ruiz, M.; Cabrillo, C.; Bermejo, F. J.; Fernandez-Perea, R.; Fischer, H. E.; Trouw, F. R. Phys. Rev. B 2000, 61, 8778.

(10) Bermejo, F. J.; Criado, A.; Fayos, R.; Fernandez-Perea, R.; Fischer, H. E.; Suard, E.; Guelylah, A.; Zuniga, J. Phys. Rev. B 1997, 56, 11536.

(11) Srinivasan, A.; Bermejo, F. J.; de Andres, A.; Dawidowski, J.; Zuniga, J.; Criado, A. Phys. Rev. B 1996, 53, 8172.

(12) Romanini, M.; Martinez-Garcia, J. C.; Tamarit, J. L.; Rzoska, S. J.; Barrio, M.; Pardo, L. C.; Drozd-Rzoska, A. J. Chem. Phys. 2009, 131, 184504.

(13) Drozd-Rzoska, A.; Rzoska, S. J.; Pawlus, S.; Tamarit, J. L. Phys. Rev. B 2006, 73, 224205 .

(14) Drozd-Rzoska, A.; Rzoska, S. J.; Pawlus, S.; Tamarit, J. L. Phys. Rev. B 2006, 74, 064201.

(15) López, D. O.; Tamarit, J. L.; de la Fuente, M. R.; Perez-Jubindo, M. A.; Salud, J.; Barrio, M. J. Phys. Condens. Matter 2000, 12, 3871.

(16) Pardo, L. C.; Lunkenheimer, P.; Loidl, A. J. Chem. Phys. 2006, 124, 124911. 
(17) Brand, R.; Lunkenheimer, P.; Schneider, U.; Loidl, A. Phys. Rev. B 2000, 62, 8878.

(18) Brand, R.; Lunkenheimer, P.; Schneider, U.; Loidl, A. Phys. Rev. Lett. 1999, 82, 1951.

(19) Brand, R.; Lunkenheimer, P.; Loidl, A. J. Chem. Phys. 2002, 116, 10386.

(20) Carpentier, L.; Decressain, R.; Descamps, M. J. Chem. Phys. 2008, 128, 024702.

(21) Hédoux, A.; Decroix, A.; Guinet, Y.; Paccou, L.; Derollez, P.; Descamps, M. J. Phys. Chem. B 2011, 115, 5746.

(22) Angell, C. A. J. Non-Cryst. Solids 1991, 131, 13.

(23) Thomas, L. H.; Welberry, T. R.; Goossens, D. J.; Heerdegen, A. P.; Gutmann, M. J.; Teat, S. J.; Lee, P. L.; C., W. C.; M., C. J. Acta Cryst. 2007, B63, 663.

(24) Cole, J. M.; Bürgi, H.; J., M. G. Phys. Rev. B 2011, 83, 224202.

(25) Ngai, K. L. Phys. Rev. E 1998, 57, 7346.

(26) Ngai, K. L. J. Phys. Condens. Matter 2002, 15, S1107.

(27) Ngai, K. L.; Lunkenheimer, P.; León, C.; Schneider, U.; Brand, R.; Loidl, A. J. Chem. Phys. 2001, 115, 1405.

(28) Kudlik, A.; Benkhof, S.; Blochowicz, T.; Tschirwitz, C.; Rössler, E. J. Mol. Struc. 1999, 479, 201.

(29) Ngai, K. L.; Paluch, M. J. Chem. Phys. 2004, 120, 857.

(30) Schneider, U.; Brand, R.; Lunkenheimer, P.; Loidl, A. Phys. Rev. Lett. 2000, 84, 5560.

(31) Pronin, A. A.; Kondrin, M. V.; Lyapin, A. G.; Brazhkin, V. V.; Volkov, A. A.; Lunkenheimer, P.; Loidl, A. Phys. Rev. E 2010, 81, 041503.

(32) Mierzwa, M.; Pawlus, S.; Paluch, M.; Kaminska, E.; Ngai, K. L. J. Chem. Phys. 2008, $128,044512$. 
(33) Casalini, R.; Roland, C. M. Phys. Rev. B 2004, 69, 094202.

(34) Aihara, A.; Kitazawa, C.; A., N. Bull. Chem. Soc. Jpn 1970, 43, 3750.

(35) Hall, P. G.; Horsfall, G. S. J. Chem. Soc., Faraday Trans. 2 1973, 69, 1071.

(36) Kolodziej, H. A.; Orzechowski, K.; Szostak, R.; Freundlich, P.; Glowiak, T.; Sorriso, S. J. Mol. Struct. 1996, 380, 15.

(37) Tan, Z. C.; Nakazawa, Y.; Saito, K.; Sorai, M. Bull. Chem. Soc. Jpn 2001, 74, 1221.

(38) Correia, N. T.; Moura Ramos, J. J.; Diogo, H. P. J. Phys. Chem. Solids 2002, 63, 1717.

(39) Moura Ramos, J. J.; Correia, N. T. Mol. Cryst. Liq. Crys. 2003, 404, 75.

(40) Shahin, M.; Murthy, S. S. N. J. Chem. Phys. 2003, 118, 7495.

(41) Shahin, M.; Murthy, S. S. N.; Singh, L. P. J. Phys. Chem. B 2006, 110, 18573.

(42) Rodríguez-Carvajal, J. FullProf Suite: Crystallographic tools for Rietveld, profile matching \& integrated intensity refinements of X-ray and/or neutron data. http: //www.ill.eu/sites/fullprof/.

(43) Groothues, H.; Kremer, F.; Collard, D. M.; Lillya, C. P. Liquid Crystals 1995, 18, 117.

(44) Elmahdy, M. M.; Floudas, G.; Mondeshki, M.; Spiess, H. W.; Dou, X.; Mllen, K. Phys. Rev. Lett. 2008, 100, 107801.

(45) Haase, N.; Grigoriadis, C.; Butt, H.; Müllen, K.; Floudas, G. J. Phys. Chem. B 2011, 115,5807 .

(46) Kremer, F.; Schönhals, A. Broadband Dielectric Spectroscopy; Springer: Berlin, 2002.

(47) Naoki, M.; Endou, H.; Matsumoto, K. J. Phys. Chem. 1987, 91, 4169. 
(48) Jain, R. K.; Simha, R. Macromolecules 1989, 22, 464.

(49) Jenau, M.; Reuter, J.; Würflinger, A.; Tamarit, J. L. J. Chem Soc. Faraday Trans. 1966, 92, 1899.

(50) Hoover, W. G.; Ross, M. Contemp. Phys. 1971, 12, 339.

(51) Salud, J.; Barrio, M.; López, D. O.; Tamarit, J. L.; Alcobé, X. J. Appl. Cryst. 1998, 31,748 . 